\title{
Selección de presas por perros callejeros en el humedal Estero Culebrón (Coquimbo, Chile)
}

\section{Prey selection by free-ranging dogs in the Estero Culebrón wetland (Coquimbo, Chile)}

\section{Víctor Bravo-Naranjo1*, Randall R. Jiménez ${ }^{2}$, Carlos Zuleta ${ }^{1}$, Jaime R. Rau ${ }^{3}$, Pablo Valladares ${ }^{4}$ \& César Piñones ${ }^{5}$}

1Departamento de Biología, Facultad de Ciencias, Universidad de La Serena, La Serena, Chile.

2Institute of Evolutionary Ecology and Conservation Genomics, University of Ulm, Ulm, Germany.

${ }^{3}$ Laboratorio de Ecología, Departamento de Ciencias Biológicas \& Biodiversidad, Universidad de Los Lagos, Osorno, Chile.

${ }^{4}$ Departamento de Biología, Universidad de Tarapacá, Arica, Chile.

${ }^{5}$ Red de Observadores de Aves y Vida Silvestre de Chile, Chile.

*Email: victor.bravon@userena.cl

\section{RESUMEN}

Examinamos los factores que influyen en la selección de presas por perros callejeros en el humedal Estero Culebrón (Coquimbo, Chile) y caracterizamos sus ataques durante 20 visitas en el año 2015. Utilizamos modelos lineales generalizados considerando el tamaño del ave, su abundancia relativa, el sitio y tipo de sustrato como variables explicativas. Registramos una jauría de siete individuos que realizaron 34 ataques sobre aves, de las cuales el pelícano presentó la mayor frecuencia de predación. El tamaño del ave y el sitio se relacionaron positivamente con los ataques. La selección del pelícano como presa podría estar relacionada con su baja distancia de inicio de vuelo y su gran tamaño, lo que facilitaría el ataque de los perros y, además, este mayor tamaño podría sostener energéticamente a la jauría, especialmente cuando es numerosa. Otras condiciones como la ausencia de totora (Typha angustipholia) y la baja profundidad del agua en el sector playa, facilitarían el desarrollo de los ataques al reducir los obstáculos a la persecución. Por último, debido al peligro que representan estos animales para las personas y la fauna de los humedales, es urgente desarrollar planes de manejo que consideren su retiro y traslado a centros de rescate, así como de implementar campañas de esterilización y educación.

Palabras clave: perros callejeros, selección de recursos, humedales urbanos, conservación de aves.

\section{ABSTRACT}

We examine the factors that influence the selection of prey by stray dogs in the Estero Culebrón wetland (Coquimbo, Chile) and characterize their attacks during 20 visits in 2015. We use generalized lineal models considering the size of the bird, its relative abundance, site and type of substrate as explanatory variables. We recorded a pack of seven individuals who carried out 34 attacks on birds, of which the pelican had the highest predation frequency. The size of the bird and the site were positively related to the attacks. The selection of the pelican as prey could be related to its low flight initiation distance and large size, which facilitates the attack by dogs and, in addition, this greater size could sustain energetically to pack of dogs, especially when they are numerous. Other conditions such the absence of cattail (Typha angustipholia) and low water depth at the beach sector, would facilitate the development of attacks by reducing obstacles to persecution. Finally, due to the danger that these animals represent for the people and the fauna of wetlands, it is urgent to develop management plans that consider the retirement and transfer of them to rescue centers, as well as to implement sterilization and education campaigns.

Keywords: free-ranging dogs, resource selection, urban wetlands, bird conservation. 


\section{INTRODUCCION}

El perro (Canis familiaris, Linneaus, 1758) es el carnívoro más abundante del mundo (Green \& Gipson 1994, Leonard et al. 2002), con una población estimada en alrededor de 700 millones de individuos (Hughes \& Macdonald 2012). Su gran abundancia se debe a que son mantenidos por el ser humano y están asociados con lugares que presentan recursos alimentarios constantes como basureros, parques o humedales (Vanak \& Gompper 2009a).

Desde su domesticación alrededor de 12.000-18000 AP (Antes del Presente, Savolainen et al. 2002, Iriarte \& Jaksic 2012), el perro ha cumplido variados roles en las comunidades humanas, tales como: mascotas, animales de trabajo, protección del ganado, vigilancia a la propiedad, asistentes de personas con discapacidad, misiones de rescate (Serpell 1996) y animales de caza (Gandiwa et al. 2013). Así de acuerdo al tipo de relación con las personas, los perros pueden clasificarse en: a) perros con dueño, b) callejeros urbanos, c) callejeros rurales, d) perros de pueblo, e) perros ferales y f) perros silvestres (Vanak \& Gompper 2009a).

Sin embargo, los perros callejeros son también un problema de conservación para la fauna silvestre, además de salud pública y seguridad para las personas (Wierzbowska et al. 2016). Estos son señalados como uno de los principales factores del declive y extinción local de varios taxa (Taborsky 1988, Banks \& Bryant 2007). Además de ocasionar lesiones y muertes sobre sus presas (Sime 1999), también actúan como competidores y vectores de enfermedades como el distemper canino, el virus de la rabia y la transmisión de diversos parásitos (Sillero-Zubiri et al. 1996, Laurenson et al. 1998, Cleaveland et al. 2000, Manor \& Saltz 2004, Campos et al. 2007, Silva-Rodríguez et al. 2010, AcostaJamett et al. 2015, Belsare \& Gompper 2015). Su presencia en los ecosistemas naturales también afectaría la riqueza y abundancia de otras especies, especialmente de las aves (Vanak \& Gomper 2009a, Iriarte \& Jaksic 2012), alterando su comportamiento (Hoopes 1993, Lenth et al. 2008), disminuyendo su detectabilidad y conductas de forrajeo (Bennet \& Zuelke 1999, Lenth et al. 2008).

Aunque la dieta de los perros callejeros se compone principalmente de vertebrados, invertebrados y plantas (Butler \& du Toit 2002, Campos et al. 2007), los perros al igual que otros carnívoros seleccionan sus presas. Esta selección está determinada por varios factores, entre los que se encuentran los costos y beneficios energéticos del proceso de caza (Creel \& Creel 2002), el tamaño y la edad de la presa (FitzGibbon \& Fanshawe 1989, Karanth \& Sunquist 1995, Radloff \& du Toit 2004), su abundancia en el lugar (Mech 1970), así como la estructura del hábitat y la estación del año
(Krüger et al. 1999, Pole et al. 2004).

En Chile, la creciente urbanización y el escaso control de la tenencia de mascotas, ha traído consigo el aumento de perros callejeros que se crían principalmente como animales comunitarios (Bonacic \& Abarca 2014), los que se desplazan hacia las zonas naturales peri-urbanas y rurales, constituyendo una amenaza constante para las personas (Gilchrist et al. 2008) y la fauna silvestre (Sime 1999). Aunque existe poca información sobre el impacto de perros callejeros sobre la vida silvestre (Weston et al. 2014) y la selección de presas, se conoce de ataques sobre lobos, tortugas e iguanas marinas, pingüinos, zorros, pumas, pudúes, conejos, ratones, coipos, coatíes, venados, huemules, vicuñas, guanacos y aves (Kruuk \& Snell 1981, Yalden \& Yalden 1990, Campos et al. 2007, García-Aguilar \& Gallo-Reynoso 2012, Corti et al. 2010, González 2010, Silva-Rodríguez \& Sieving 2011, Aliaga-Rossel et al. 2012, CONAF 2012, Iriarte \& Jaksic 2012). Debido a que el humedal Estero Culebrón ha concitado una creciente preocupación por su conservación, y puesto que la avifauna es uno de los principales componentes de su biodiversidad, nos propusimos documentar los ataques y determinar los factores que influyen en la selección de aves como presas por los perros callejeros que habitan en el sector.

\section{MATERIALES Y MÉTODOS}

\section{ZONA DE ESTUDIO}

El humedal Estero Culebrón (UTM 275942-6683177) está situado a 1,5 km de la caleta de Coquimbo, Chile (Fig. 1), ciudad donde se ha estimado una densidad de 2380 perros/ $\mathrm{km}^{2}$ (Acosta-Jamett et al. 2010). Las aguas de este pequeño humedal (18 ha de superficie), se originan en el estero que lo alimenta y el mar (Figueroa et al. 2009). El Estero Culebrón, es un humedal urbano y está considerado como sitio prioritario para la conservación de la biodiversidad (CONAMA 2003). Su flora está representada principalmente por Brea (Tessaria absinthioides, Ruiz et Pavón 1753), Hierba sosa (Sarcocornia fruticosa, Scott 1978), Totora (Typha angustipholia, Linnaeus 1753) y Junquillo (Juncus acutus, Linnaeus 1753) (Luna 2005), y en cuanto a la avifauna, registra una riqueza estimada de alrededor de 124 especies (eBird 2016).

\section{Muestreo}

Se realizó un total de 20 muestreos durante el año 2015 , desarrollados entre las 8:00 y 12:00 AM. Estos, fueron llevados a cabo durante fines de semana sucesivos desde que se registró el primer ataque de perros sobre aves silvestres (segunda semana de enero). 


\section{Riqueza de Aves}

La riqueza específica de aves del humedal Estero Culebrón se estimó con el estimador no paramétrico de Jackknife de segundo orden (Burnham \& Overton 1978) y la comparamos con la riqueza observada para determinar el nivel de alcance y precisión de nuestro inventario. Estos cálculos fueron realizados con el programa "Species Diversity and Richness IV" (Seaby \& Henderson 2006).

DESCRIPCIÓN DE VARIABLES PARA EL ANÁLISIS DE SELECCIÓN DE PRESAS Para el estudio de selección de presas definimos una variable respuesta binaria, como presencia o ausencia de un ataque por perros callejeros a aves. La presencia de un ataque se definió como todas aquellas conductas que constituyeron persecución de las aves, independientemente si éstas fueron exitosas o fallidas. Cada vez que se registró un ataque, se examinaron los factores (covariables): sitio, sustrato, tamaño de la presa y abundancia relativa de cada especie (ver Tabla 1). La ausencia de ataque se definió como todos aquellos comportamientos donde la jauría se encontró realizando actividades de cualquier otro tipo (i.e. descanso, juegos). Durante los muestreos en que no observamos ataques, y cada 40 minutos, se registraron las aves presentes, su abundancia relativa y las demás covariables (sitio y sustrato). Después se seleccionó una especie (y su correspondiente tamaño corporal) hasta completar el mismo número de casos de ataques (34). Estos datos se ingresaron a través de una matriz a Lenguaje estadístico R (R Core Team 2015) para su posterior análisis. La selección al azar se desarrolló mediante la función sample de $\mathrm{R}$, para muestras con reemplazo, es decir, que la misma especie podría volverse a seleccionar, tal y como ocurre en los casos de ataques.

El conjunto de variables explicativas utilizado en este estudio fue seleccionado de acuerdo a lo señalado en la literatura (e.g. Raldoff \& Du toit 2004, Roley et al. 2010), porque éstas no son ambiguas y son fácilmente cuantificables en el campo. Para el tamaño de las presas, se consideró la longitud del ave según lo descrito en Jaramillo (2011). Para la abundancia relativa, se multiplicó la abundancia de la especie por 100 y se dividió por el total de aves observadas en el día de muestreo. El sitio correspondió a dos sectores del humedal: interior y playa (Fig. 1), diferenciados por la cercanía a la línea de costa. Por último, el sustrato se clasificó de acuerdo con las características del suelo: con agua superficial, y con vegetación (Tabla 1).

\section{ANÁLISIS DE LOS DATOS}

Para determinar qué factores influyeron en la probabilidad de ataque de perros sobre las aves se utilizó la aproximación de teoría de la información (information-theoretic approach, Burnham \& Anderson 2002). Para ello, se ajustaron modelos lineales generalizados con distribución binomial con la presencia (1) o ausencia (0) de ataques de perros a las aves como variable respuesta, y todas las posibles combinaciones de las variables explicativas. Además, se ajustó un modelo nulo para evaluar si existió o no relación de los ataques con las variables seleccionadas. En este sentido, si el modelo que mejor explicó los ataques fue el modelo nulo, implicaría que i) las variables consideradas no fueron las que podrían explicar la selección de presas o ii) los perros no seleccionaron a las aves. Posteriormente, la selección del modelo con mejor ajuste a los datos se realizó a partir del Criterio de Información de Akaike corregido para muestras pequeñas (AIC ${ }_{c}$, Burnham \& Anderson 2002), donde el modelo con el menor valor de AIC se consideró como el modelo con mejor ajuste a los datos observados en relación con los otros modelos candidatos. Además, se calculó la diferencia entre el mejor modelo y el más próximo $(\triangle A I C)$, y el peso de $\operatorname{AIC}_{c}\left(\mathrm{AIC}_{c} w\right)$ para determinar el apoyo relativo del mejor modelo respecto de los modelos candidatos (Burnham \& Anderson 2002).

TABLA 1. Descripción de las variables incluidas en los modelos para explicar la selección de presas por perros callejeros en el humedal Estero Culebrón. / Description of variables included in the models to explain the prey selection by free-ranging dogs in the Estero Culebrón Wetland.

\begin{tabular}{lll}
\hline Variable & Descripción & Resumen de la variable \\
\hline Tamaño de la presa & Continua & Entre 12 y $127 \mathrm{~cm}$ de longitud \\
Abundancia relativa & Continua & Entre 0,2 y 57 \\
Sitio & Categórica & $\begin{array}{l}\text { Interior: correspondiente al sur desde la carretera } \\
\text { Playa: al norte de la carretera }\end{array}$ \\
Sustrato & Categórica & $\begin{array}{l}\text { Agua superficial: permitía la marcha de los perros } \\
\text { Vegetación: área cubierta por brea, hierba sosa y totora }\end{array}$ \\
\hline
\end{tabular}


Debido a que no hubo un único modelo que de manera clara fuera superior comparado a los otros (i.e. $\Delta \mathrm{AlCc}>2$ or $\left.\mathrm{AIC}_{\mathrm{c}} w>0,9\right)$ se procedió a realizar un promediado de modelos basado en aquellos que estuvieran dentro del set de modelos a un $95 \%$ de confianza (Burnham \& Anderson 2002, Symonds and Moussalli 2011). Este set de modelos con $95 \%$ de confianza comienza con el modelo con mayor $\mathrm{AIC}_{\mathrm{c}}$ $w$ y se van añadiendo el siguiente modelo con mayor $\mathrm{AIC}_{c} w$ hasta que la suma acumulativa exceda 0,95. El promediado del modelo se realizó con el paquete "MuMIN" de R, y se obtuvieron los coeficientes promedio, errores estándar, intervalos de confianza al 95\% (IC 95\%) e importancia relativa de las variables explicativas. La importancia relativa de las variables explicativas se utiliza para clasificar dichas variables en términos de su jerarquía para explicar un efecto, y ésta se obtiene sumando los $\mathrm{AICc} w$ de los modelos donde una variable $j$ estuvo presente. Aquellas variables explicativas que obtuvieron una importancia relativa $>0,8$ o IC $95 \%$ que no incluyeran el 0 fueron consideradas importantes (Symonds and Moussalli 2011). Se utilizó el modelo que contuviera solo las variables consideradas importantes para graficar su relación con la probabilidad de ataque. El modelaje estadístico se realizó en R usando los paquetes "AICcmodavg", "MuMIN", "visreg" y "ggplot2".

\section{RESULTADOS}

Durante la campaña, se registró una jauría compuesta por siete individuos ( $95 \% \mathrm{IC}=6-7$ ) y un total 37 especies de aves $(95 \%$ IC $=18-23)$ en el humedal, riqueza que coincidió con lo esperado según el estimador de Jackknife. De 34 ataques de perros registrados en el Estero Culebrón, sólo se observaron ataques sobre las aves en los primeros 14 días de muestreo, los que variaron entre 1 y 4 ataques por día, no registrándose más ataques en las campañas posteriores. Sólo se observaron
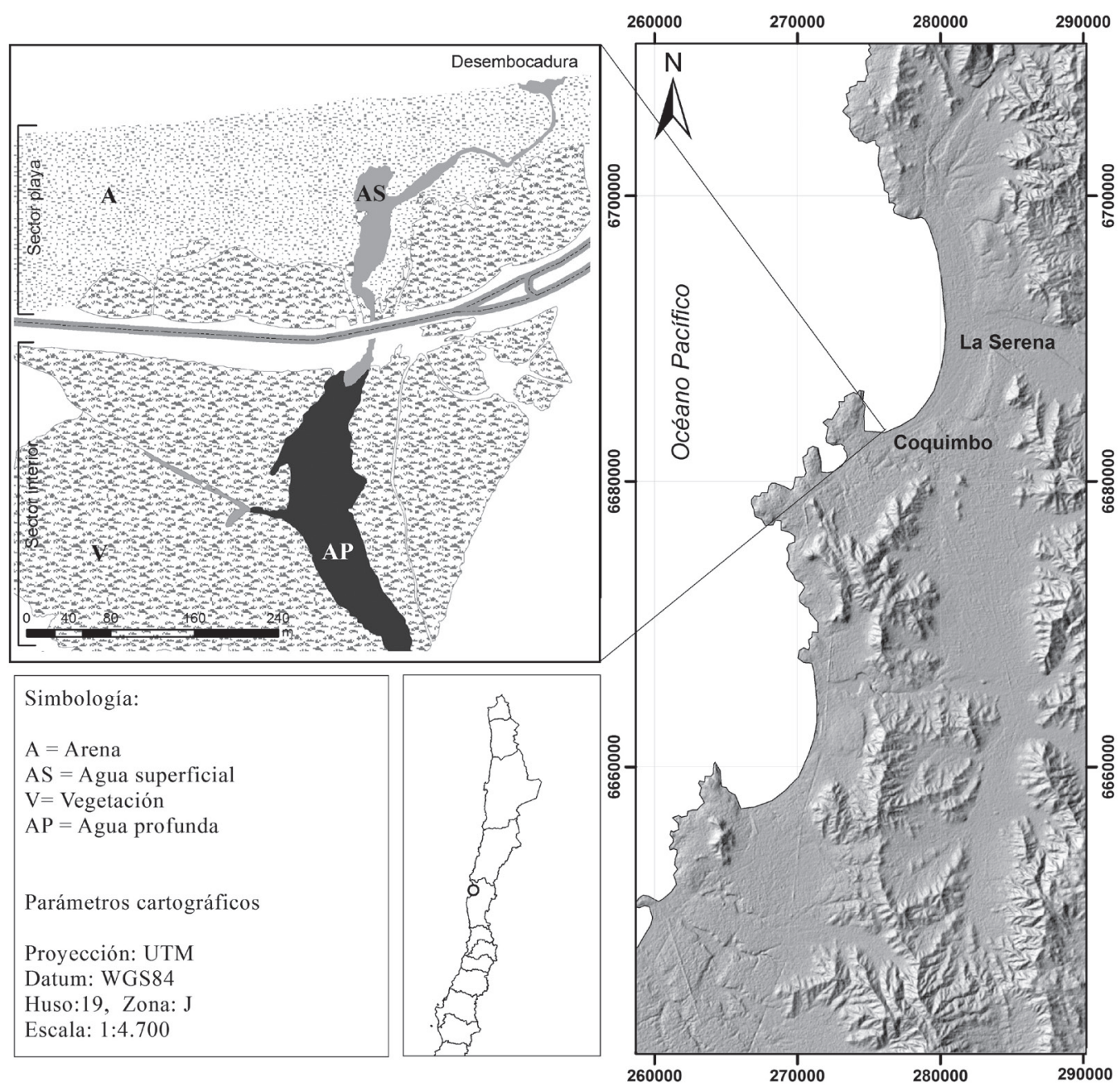

Figura 1. Localización general del humedal Estero Culebrón en la Región de Coquimbo, Chile. / General location of Estero Culebrón Wetland in the Coquimbo Region, Chile. 
perros mestizos, y sólo uno de raza pastor alemán (Fig. 3). La jauría estaba constituida por perros de gran tamaño y su composición se mantuvo constante, con un líder que exhibía conductas de mayor agresividad y de aparente coordinación de los ataques.

Los perros seleccionaron nueve especies de aves como potenciales presas de las 37 especies que se encontraron en el humedal (24,3\%). El pelícano (Pelecanus thagus, Molina 1782) fue la especie con mayor frecuencia de caza con 24 ataques (71\%), seguido del yeco (Phalacrocorax brasilianus, Gmelin 1989) con dos ataques (6\%), al igual que la garza chica (Egretta thula, Molina 1782). Otras especies como la tagua común (Fulica armillata, Vieillot 1817), garza boyera (Bubulcus ibis, Linné 1758), gaviota dominicana (Larus dominicanus, Lichtenstein 1823), pato jergón grande (Anas georgica, Gmelin 1789), pilpilén común (Haematopus palliatus, Murphy 1925) y cormorán lile (Phalacrocorax gaimardi, Lesson \& Garnot 1828) registraron sólo un ataque.

Según el modelo promediado, la probabilidad de ataques por perros callejeros se puede explicar por el tamaño de la presa y el sitio de ataque (ICs $95 \%$ de las covariables no traslapan el 0 y su importancia relativa fue $>0,8$ ) (Tabla 3 ). Los coeficientes del modelo promediado y la predicción del modelo con las variables más influyentes indicaron que el tamaño de presa presenta una relación positiva con un evento de ataque, y en la zona de playa existe una probabilidad mayor de ataque comparado con el interior del humedal (Tabla 3, Fig. 2). En este sentido, a medida que aumentaba en un centímetro el tamaño del ave, también la probabilidad de ataque incrementaba en un $5 \%$, y si además las aves se encontraban en la playa, la probabilidad que se produzcan ataques de perros en esta zona, aumentaba en un $39 \%$ comparado con el interior del humedal.

En cuanto al desarrollo de los ataques, se observó que en general a) la jauría de perros se acercaba sigilosamente y sólo avanzaba cuando el líder se desplazaba. El líder fue quien más se acercó a la presa, y b) el comienzo del ataque coincidía con el momento cuando el ave, al sentirse incómoda, preparaba el vuelo. El líder es quien se lanzaba al ataque y tomaba a la presa seguido inmediatamente por el resto del grupo (Fig. 3), que luego se desplazaban hacia el interior del humedal. La jauría también utilizó la playa del humedal como área de reposo y acoso sobre varias especies de aves, principalmente de gaviota dominicana ( $L$. dominicanus), gaviota de Franklin (Leucocephalus pipixcan, Wagler 1831), gaviotín elegante (Thalasseus elegans, Gambel 1849) y zarapito (Numenius phaeopus, Linné 1758).
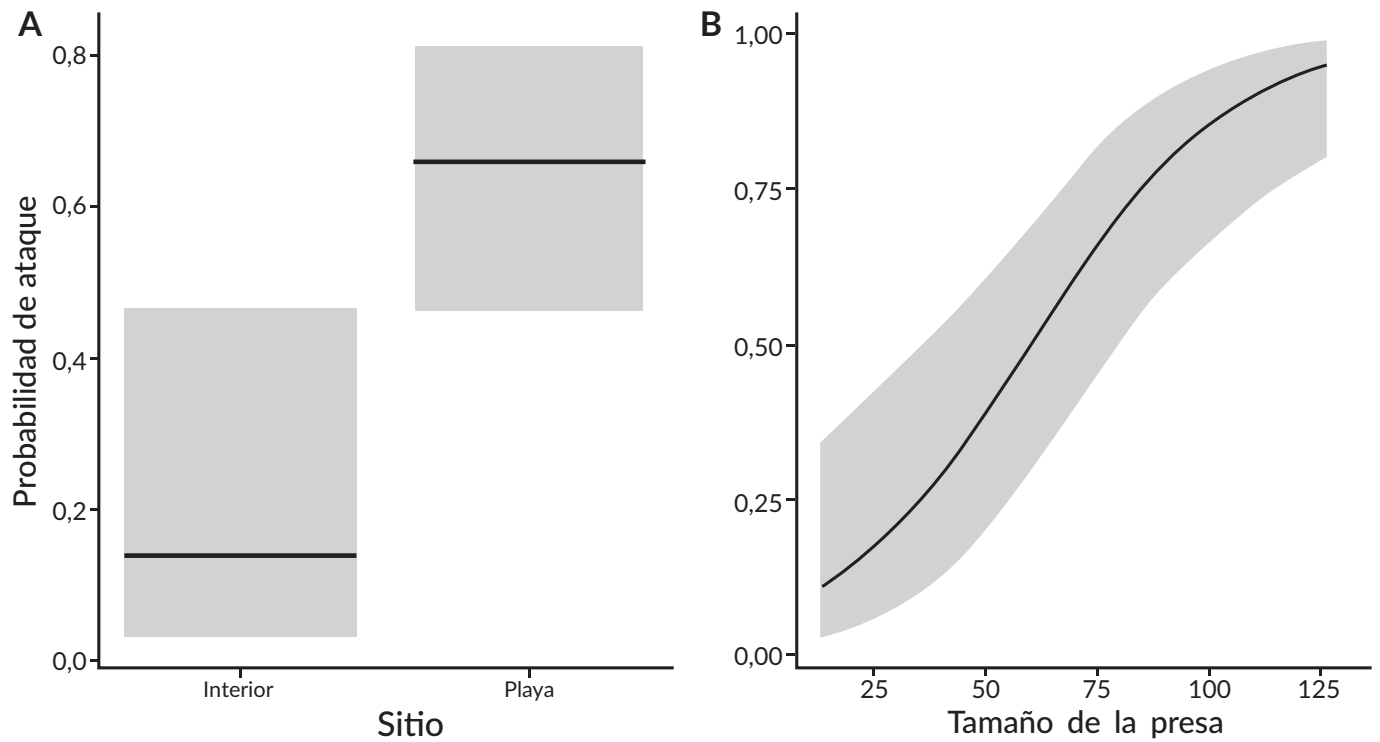

Figura 2. Relaciones entre la probabilidad de ataque a las aves del humedal Estero Culebrón por jaurías de perros de acuerdo a: el sitio de ataque (A) y el tamaño de la presa (B) (mientras se establecen todas las demás covariables en sus niveles medios (variables continuas) y con la categoría más común (variables categóricas), derivada del modelo que contiene solo las covariables muy influyentes. La línea negra representa el ajuste y la barra gris el intervalo de confianza al 95\%. / Relationships between the probability of attacking on birds in Estero Culebrón wetland by dog packs according to: the attack site (A) and the size of the prey (B) (while all other covariates are established at their average levels (continuous variables) and with the most common category (categorical variables), derived from the model that contains only very influential covariates: the black line represents the adjustment line and the gray line the $95 \%$ confidence interval. 
TABLA 2. Modelos candidatos, número de parámetros (K), Valor de Akaike para muestras pequeñas (AICc), diferencia entre el modelo actual y el mejor modelo $(\triangle \mathrm{AIC}$ ) y peso (AICcW) de los modelos ajustados. Los modelos en negrita conforman el set de modelos con $95 \%$ de confianza. / Candidate models, number of parameters (K), Akaike value for small samples (AICc), difference between the current model and the best model $(\triangle \mathrm{AIC})$ and weight $\left(\mathrm{AIC}_{\mathrm{c}} \mathrm{w}\right)$ of the adjusted models. The bold models make up the set of models with $95 \%$ confidence.

\begin{tabular}{lcccc}
\hline Modelos candidatos & K & AICc & $\Delta$ AICc & AIC $_{c} w$ \\
\hline 1. Tamaño + Sitio & 3 & 51,76 & 0,00 & 0,49 \\
2. Tamaño + Sustrato + Sitio & 4 & 53,64 & 1,87 & 0,19 \\
3. Tamaño + Sitio + Abundancia relativa & 4 & 54,00 & 2,24 & 0,16 \\
4. Tamaño + Sustrato + Sitio + Abundancia relativa & 5 & 55,95 & 4,19 & 0,06 \\
5. Tamaño + Sustrato & 3 & 56,28 & 4,52 & 0,05 \\
6. Tamaño & 2 & 57,71 & 5,95 & 0,02 \\
7. Tamaño + Sustrato + Abundancia relativa & 4 & 58,47 & 6,71 & 0,02 \\
8. Tamaño + Abundancia relativa & 3 & 59,63 & 7,86 & 0,01 \\
9. Sustrato + Sitio & 3 & 70,14 & 18,38 & 0,00 \\
10. Sustrato+ Sitio + Abundancia relativa & 4 & 70,57 & 18,81 & 0,00 \\
11. Sustrato & 2 & 71,05 & 19,29 & 0,00 \\
12. Sitio + Abundancia relativa & 3 & 73,78 & 22,02 & 0,00 \\
13. Sitio & 2 & 74,56 & 22,80 & 0,00 \\
14. Abundancia relativa & 2 & 79,07 & 27,30 & 0,00 \\
15. Modelo nulo & 1 & 82,48 & 30,72 & 0,00 \\
\hline
\end{tabular}

Modelos clasificados por el valor de AICc / Models-ranked by AICc value.

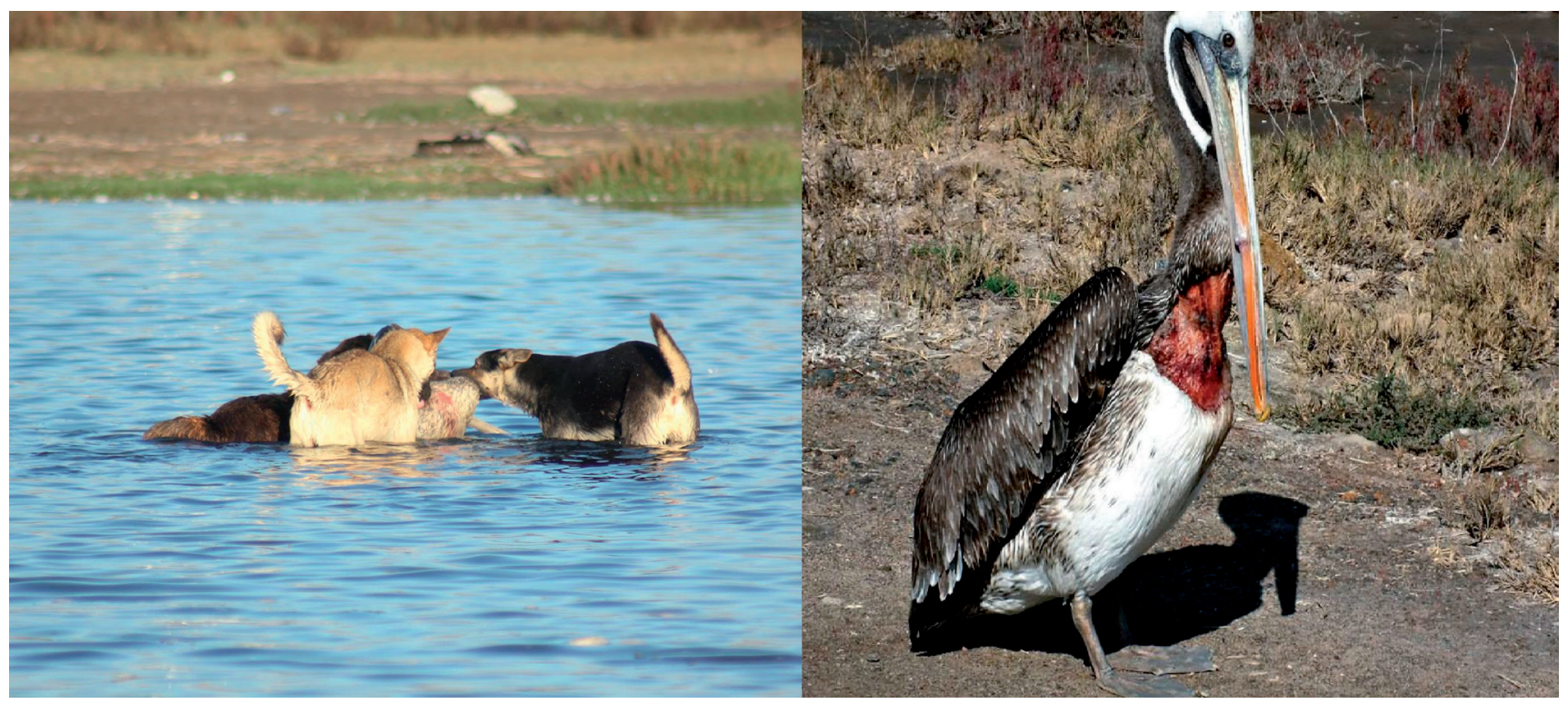

Figura 3. Perros atacando en grupo un pelícano (izquierda) y pelícano con una laceración grave producto del ataque de perros (derecha, foto: Lorena Toro) en el humedal Estero Culebrón. / Dogs attacking pelican in group (left) and pelican with a severe laceration product of dogs attack (right, photo: Lorena Toro) in the Estero Culebrón wetland. 
TABLA 3. Parámetros del modelo promediado del set de modelos con 95\% de confianza (ver Tabla 2), Error estándar ajustado (ES), intervalos de confianza (IC 95\%) e importancia relativa. / Parameters of the averaged model of the model set with 95\% confidence (see Table 2), Adjusted standard error (ES), confidence intervals $(95 \% \mathrm{CI})$ and relative importance.

\begin{tabular}{lccccc}
\hline \multicolumn{1}{c}{ Parámetro } & $\begin{array}{c}\text { Coeficiente } \\
\text { Promediado }\end{array}$ & ES & IC 95\% inferior & IC 95\% superior & $\begin{array}{c}\text { Importancia } \\
\text { relativa }\end{array}$ \\
\hline Tamaño de presa & 0,04 & 0,01 & 0,02 & $0,06 c$ & 1,00 \\
Sitio de ataque (Playa) & 2,37 & 1,03 & 0,35 & $4,39 c$ & 0,95 \\
Sustrato (Vegetación) & $-0,71$ & 0,91 & $-2,49$ & 1,08 & 0,32 \\
Abundancia relativa & 0,07 & 0,27 & $-0,46$ & 0,61 & 0,23 \\
\hline
\end{tabular}

'Variables con intervalos de confianza al 95\% (IC 95\%) que no traslapan el 0

\section{DISCUSIÓN}

Los perros callejeros observados en el Estero Culebrón, al igual que el perro salvaje africano (Lycaon pictus, Temminck 1820), cazan socialmente y mantienen una estructura social similar a otros cánidos (Creel \& Creel 1995, Cafazzo et al. 2010). Las estrategias de ataques registradas coinciden con otras observaciones realizadas en el lugar, pero difieren de las estrategias de caza observadas para ataques de perros sobre mamíferos (ver Lowry \& McArthur 1978). Para cánidos nativos con requerimientos similares a los perros callejeros, como el lobo (Canis lupus, Linnaeus 1758), se ha encontrado que sus ataques ocurren frecuentemente en áreas donde la vegetación es más densa (Husseman et al. 2003), al igual como ocurre con otros carnívoros (Mills et al. 2004). En cambio, en el estero El Culebrón ocurre lo contrario, puesto que la mayor frecuencia de ataques fue en el sitio playa, que se caracterizó por su escasa vegetación y por presentar dominancia en especies vegetales de baja altura como grama salada (Distichlis spicata, L Greene 1887) y cristaria (Cristaria glaucophylla, José de Cavanilles 1799), factores que facilitarían la rapidez de ataque de la jauría.

Los perros callejeros son depredadores generalistas y oportunistas, mostrando pocas diferencias en el consumo de presas (Butler et al. 2004, Campos et al. 2007, Vanak \& Gomper 2009b, Silva-Rodríguez et al. 2010). Sin embargo, en el Estero Culebrón, los perros prefieren depredar a las aves de mayor tamaño como el pelícano. Además, los resultados sugieren que la mayor abundancia de una presa no es indicativa de una mayor probabilidad de ataque. En este sentido, la selección de presas podría explicarse también porque el pelícano tiene una distancia de iniciación de vuelo relativamente corta (Blumstein et al. 2003), lo que permite a los perros disminuir su distancia para comenzar un ataque. Además, tanto para el pelícano como para otras aves, se desconoce si estas logran percibir a los perros como potenciales depredadores (Lima \& Bednekoff 1999).

Otro factor a considerar, sería el mayor tamaño del pelícano comparado con otras aves del humedal. Una presa de mayor tamaño podría sostener energéticamente a la jauría, disminuyendo los esfuerzos de caza, como ha sido documentado en perros "ferales" (Davies-Mostert et al. 2013). De hecho, el tamaño de la presa y el sitio playa, obtuvieron una mayor importancia relativa que otras variables exploratorias (Tabla 3), con un fuerte ajuste para explicar la selección de presas por perros callejeros en este humedal, contrario a lo que sucedió con el sustrato y la abundancia relativa de aves (importancia relativa $<0,8$ ).

En el humedal urbano El Culebrón se ha documentado una relación negativa entre la presencia de perros y la riqueza de aves en la temporada invernal (Chávez-Villavicencio et al. 2015), pero no se ha demostrado sus efectos sobre la abundancia de aves, especialmente de aquellas con preferencia de caza. Los perros tendrían un impacto significativo sobre la avifauna, como ha ocurrido en otros lugares con presencia de especies exóticas y patógenos (Wikelski et al. 2004). Además, como los perros tienden a cazar por entretención, esta situación podría culminar con la extinción local de algunas especies de aves con problemas de conservación como el playero ártico (Calidris canutus, Linnaeus 1758) y el cuervo de pantano (Plegadis chihi, Vieillot 1817), especies clasificadas En Peligro y Casi Amenazado (MMA 2016, IUCN 2016) y En Peligro Crítico (Servicio Agrícola y Ganadero 2015, MMA 2016), respectivamente.

El Estero Culebrón es uno de los humedales costeros más amenazados de la Región de Coquimbo (Luna 2005) y sus 
poblaciones de aves soportan un gran número de presiones que coinciden con el ciclo reproductivo de varias especies. Nuestros datos demuestran en este sitio la depredación de aves por perros callejeros. Sin embargo, se desconoce del consumo completo de las presas del pelícano y otras aves (Fox 1971, Coppinger \& Coppinger 2001). Aunque $P$. thagus no presenta problemas de conservación (SAG 2015), globalmente se considera Casi Amenazado y en el país se desconoce el estado actual de sus poblaciones (Cursach et al. 2018). Así, existe una amenaza creciente a la biodiversidad del humedal por las muertes a la avifauna ocasionadas por los ataques de perros, además de ocasionar un problema de salud pública (Gilchrist et al. 2008), que se suma al riesgo de ataques a observadores de aves, veraneantes, deportistas, estudiantes y otros visitantes del humedal.

Pese a que se reconoce la importancia de los humedales urbanos para la mantención de la biodiversidad y la amenaza que representan la presencia de perros callejeros en estos ecosistemas, aún no se desarrollan las estrategias de manejo y conservación adecuadas; Molina (2015) presentó buenas orientaciones al respecto. Debido a lo anterior y considerando la alta abundancia de perros callejeros en Coquimbo (AcostaJamett et al. 2010) que favorece la migración de nuevos individuos a las áreas silvestres y rurales (Iriarte \& Jaksic 2012), la amenaza que se cierne sobre las aves es constante.

Recomendamos que se implementen en El Culebrón las sugerencias de Molina (2015) y el protocolo de manejo de perros desarrollado por "The Royal Society for the Prevention of Cruelty to Animals" (RSPCA). En este sentido, retirar los perros callejeros del humedal y trasladarlos a un centro de rescate; así como aumentar las campañas de esterilización para perros domésticos y callejeros, sobre todo de las áreas fuente como la caleta de Coquimbo, son medidas de corto plazo que pueden contribuir efectivamente a disminuir los ataques de los perros a la avifauna nativa. Sin embargo, estas medidas deben ser complementadas con campañas recurrentes de tenencia responsable de mascotas, coordinación de los agentes públicos pertinentes y con la educación de la ciudadanía para la valoración y conservación de los humedales urbanos.

\section{AGRADECIMIENTOS}

Los autores agradecen al Fondo de Protección Ambiental (FPA NAC-I-032-2014) del Ministerio del Medio Ambiente y al Proyecto Sustentabilidad \& Sistemas Naturales de Chile central DIDULS (2018-2020) por el financiamiento de esta investigación. Esta publicación se genera en el marco colaborativo de la Red de investigación en Sustentabilidad de las Universidades del Estado de Chile (Convenio Marco Objetivo en Red 1756-1856).

\section{REFERENCIAS}

Acosta-Jamett, G., Cleaveland, S., Cunningham, A.A., deC. BronsVOORT, B.M. 2010. Preventive Veterinary Medicine 94(3-4): 272-281. DOI: 10.1016/j. prevetmed.2010.01.002

Acosta-Jamett, G., Surot, D., Cortés, M., Marambio, V., Valenzuela, C., Vallverdu, A., Ward, M.P. 2015. Epidemology of canine distemper and canine parvovirus in domestic dogs in urban and rural areas of the Araucanía region in Chile. Veterinary Microbiology 178(3-4): 260-264. DOI: 10.1016/j.vetmic.2015.05.012

Aliaga-Rosel, E., Ríos-Uzeda, B., Ticona, H. 2012. Amenazas de perros domésticos en la conservación del cóndor, el zorro y el puma en las tierras altas de Bolivia. Revista Latinoamericana de Conservación 2(2): 78-81.

Banks, P.B., Bryant, J.V. 2007. Four-legged friend or foe? Dog walking displaces native birds from natural areas. Biology Letters 3(6): 611-613. DOI: 10.1098/rsbl.2007.0374

Belsare, A.V., Gomprer, M.E. 2015. To vaccinate or not to vaccinate: lessons learned from an experimental mass vaccination of free-ranging dog populations. Animal Conservation 18(3): 219-227. DOI: 10.1111/acv.12162

Bennet, K., ZuelKe, E. 1999. The effects of recreation on birds: A literature review. Unpublished report. Delaware Natural Heritage Program. USA. 17 pp.

Blumstein, D.T,. Anthony, L.L., Harcourt, R.G., Ross, G. 2003. Testing a key assumption of wildlife buffer zones: is flight initiation distance a species-specific trait?. Biological Conservation 110(1): 97-100. DOI: 10.1016/S0006-3207(02)00180-5

Bonacic, C., AbarCa, K. 2014. Hacia una política y legislación para el control de poblaciones de cánidos y calidad de vida de las personas: un enfoque multidisciplinario. Temas de la Agenda Pública N65. Pontificia Universidad Católica de Chile, Santiago. 11 pp.

Boyce, M.S. 2006. Scale for resource selection functions. Diversity and Distributions 12(3): 269-276. DOI: 10.1111/j.13669516.2006.00243.x

Burnham, K., Anderson, D.R. 2002. Model Selection and Multimodel Inference: A Practical Information-Theoretic Approach, Springer-Verlag, New York, USA. 488 pp.

Burnham, K.P., Overton, W.S. 1978. Estimation of the size of a closed population when capture probabilities vary among animals. Biometrika 65(3): 623-633. DOI: $10.2307 / 2335915$ 
Butler, J.R., Du ToIT, J.T. 2002. Diet of free-ranging domestic dogs (Canis familiaris) in rural Zimbabwe: implications for wild scavengers on the periphery of wildlife reserves. Animal Conservation 5(1): 29-37. DOI: 10.1017/ S136794300200104X

Butler, J.R., Du ToIt, J.T., BINGHAM, J. 2004. Free-ranging domestic dogs (Canis familiaris) as predators and prey in rural Zimbabwe: Threats of competition and disease to large wild carnivores. Biological Conservation 115(3): 369-78. DOI: 10.1016/S0006-3207(03)00152-6

Cafazzo, S., Valsecchi, P., Bonanni, R., Natoli, E. 2010. Dominance in relation to age, sex, and competitive contexts in a group of free-ranging domestic dogs. Behavioral Ecology 21(3): 443-455. DOI: 10.1093/beheco/arq001

Calcagno, V., de Mazancourt, C. 2010. glmulti: An R Package for Easy Automated Model Selection with (Generalized) Linear Models. Journal of Statistical Software 34(12): 1-29. DOI: 10.18637/jss.v034.i12

Campos, C.B., Esteves, C.F., Ferraz, K.M.P.M.B., Crawshaw JR, P.G., VERDADE, L.M. 2007. Diet of free-ranging cats and dogs in a suburban and rural environment, south-eastern Brazil. Journal of Zoology 273(1): 14-20. DOI: 10.1111/j.14697998.2007.00291.x

Chávez-Villavicencio, C., Molina-Pérez, P., Tabilo-Valdivieso, E. 2015. Respuesta de la riqueza de aves en presencia de visitantes, vehículos y perros en el humedal "El Culebrón", Chile. The Biologist (Lima) 13(2): 313-327.

Cleaveland, S., Appel, M.G.J, Chalmers, W.S.K, Chillingworth, C., KaARE, M., Dye, C. 2000. Serological and demographic evidence for domestic dogs as a source of canine distemper virus infection for Serengeti wildlife. Veterinary Microbiology 72(3-4): 217- 227. DOI: 10.1016/S03781135(99)00207-2

CONAF. 2012. Análisis de la ocurrencia de ataques de perros y gatos a fauna silvestres protegida en el Sistema Nacional de Áreas Silvestres Protegidas del Estado 2007-2012. Corporación Nacional Forestal, Santiago. 7 pp.

CONAMA. 2003. Estrategia Nacional de Biodiversidad. Comisión Nacional del Medio Ambiente \& Ocho libros Editores Ltda. Santiago. 637 pp.

Coppinger, R., Coppinger, L. 2001. Dogs: A Startling New Understanding of Canine Origin, Behavior \& Evolution. Scribner. University of Chicago Press, New York. 352 pp.

Corti, P., Wittmer, H.U., Festa-Bianchet, M. 2010. Dynamics of a small population of endangered huemul deer (Hippocamelus bisulcus) in Chilean Patagonia. Journal of Mammalogy 91(3): 690-697. DOI: 10.1644/09-MAMM-A-047.1

CreEL, S., CreEL, N. 1995. Communal hunting and pack size in African wild dogs, Lycaon pictus. Animal Behaviour 50(5):
1325-1339. DOI: 10.1016/0003-3472(95)80048-4

Cursach, J.A., Rau, J.R., Gelcich, S., Rodríguez-Maulén, J. 2018. Situación poblacional del pelícano peruano (Pelecanus thagus) en Chile: prospección inicial. Ornitología Neotropical 29: 77-89.

Davies-Mostert, H.T., Mills, M.G.L., Macdonald, D.W. 2013. Hard boundaries influence African wild dogs diet and prey selection. Journal of Applied Ecology 50(6): 1358-1366. DOI: 10.1111/1365-2664.12129

Dolores U, M., Militino, A.F., Arnholt, A.T. 2016. Probability and Statistics with R. Second Edition. CRC Press, New York. $983 \mathrm{pp}$.

EBIRD. 2016. Una base de datos en línea para la abundancia y distribución de las aves. Ithaca, New York. URL: http:// www.ebird.org. Accedido: Mayo 06, 2015.

Figueroa, R., Suarez, M.L., Andreu, A., Ruiz, V.H., Vidal-Abarca, M.R. 2009. Caracterización ecológica de humedales de la zona semiárida en Chile central. Gayana 73(1): 76-94. DOI: 10.4067/S0717-65382009000100011

FitzGibbon, C.D., Fanshawe, J.H. 1989. The condition and age of Thomson's gazelles killed by cheetahs and wild dogs. Journal of Zoology 218(1): 99-107. DOI: 10.1111/j.14697998.1989.tb02528.x

Fox, M.W. 1971. Behaviour of Wolves, Dogs and Related Canids. Harper Collins, New York. 217 pp.

Gandiwa, E., Heitkönig, I.M.A., Lokhorst, A.M., Prins, H.H.T., LeEUWIS, C. 2013. Illegal hunting and law enforcement during a period of economic decline in Zimbabwe: A case study of northern Gonarezhou National Park and adjacent areas. Journal of Nature Conservation 21(3): 133-142. DOI: 10.1016/j.jnc.2012.11.009

García-Aguilar, M., Gallo-Reynoso, J.P. 2008. Perros ferales en la isla de Cedros, Baja California, México: una posible amenaza para los pinnípedos. Revista Mexicana de Biodiversidad 83(3): 785-789. DOI: 10.7550/rmb.24851

Gilchrist, J., SACKS, J.J., White, D., Kresnow, M.J. 2008. Dogs bite: still a problem?. Injury Prevention 14(5): 296-301. DOI: 10.1136/ip.2007.016220

GonzÁLEZ, B.A. 2010. ¿Qué problemas de conservación tienen las poblaciones de guanaco en Chile?. Ambientes Forestales 9: 28-38.

Green, J.S., Gipson, P.S. 1994. Feral Dogs. En: Hygnstrom, S.E., Tim, R.M., Larson, G.E. (Eds) Prevention and Control of Wildlife Damage: c77-c81. University of NebraskaLincoln, Nebraska.

Hoopes, E.M. 1993. Relationships between human recreation and piping plover foraging ecology and chick survival. M.S. Thesis. University of Massachusetts, Massachusetts, USA.

Hughes, J., MACDONALD, D.W. 2012. A review of the interactions between free-roaming domestic dogs and wildlife. 
Biological Conservation 157: 341-351. DOI: 10.1016/j. biocon.2012.07.005

Husseman, J.S., Murray, D.L., Power, G., Mack, C., Wenger. C.R., QuigLEY, H. 2003. Assessing differential prey selection patterns between two sympatric large carnivores. Oikos 101(3): 591-601. DOI: 10.1034/j.16000706.2003.12230.x

IRIARTE, A., JAKSIC, F. 2012. Los Carnívoros de Chile. Ediciones Flora y Fauna Chile y CASEB. Pontificia Universidad Católica de Chile, Santiago. $260 \mathrm{pp}$.

IUCN. 2016. IUCN Red List of Threatened Species. International Union for Conservation of Nature. URL: http://www. iucnredlist. Accedido: Junio 21, 2016.

Jaramillo, A. 2011. Aves de Chile. Lynx Ediciones, Barcelona. 240 pp.

JoHNSON, D.H. 1980. The comparison of usage and availability measurements for evaluating resource preference. Ecology 61(1): 65-71. DOI: 10.2307/1937156

Karanth, K.U., Sunquist, M.E. 1995. Prey selection by tiger, leopard and dhole in tropical forests. Journal of Animal Ecology 64(4): 439-450. DOI: 10.2307/5647

KRÜGER, S.C., LAWES, M.J., MadDoCK, A.H. 1999. Diet choice and capture success of wild dog (Lycaon pictus) in HluhluweUnfolozi Park, South Africa. Journal of Zoology 248(4): 543-551. DOI: 10.1111/j.1469-7998.1999.tb01054.x

KruUK, H., SNel, H. 1981. Prey selection by feral dogs from a population of marine iguanas (Amblyrhynchus cristatus). Journal of Applied Ecology 18(1): 197-204. DOI: $10.2307 / 2402489$

Laurenson, K., Sillero-Zubiri, C., Thompson, H., Shiferaw, F., ThiRgood, S., Malcolm, J. 1998. Disease threats to endangered species; Ethiopian wolves, domestic dogs and canine pathogens. Animal Conservation 1(4): 273280. DOI: 10.1111/j.1469-1795.1998.tb00038.x

Lenth, B.E., KniGht, R.L., Brennan, M.E. 2008. The effects of dog on wildlife communities. Natural Areas Journal 28(3): 218-227. DOI: $10.3375 / 0885-8608(2008) 28$ [218:TEODOW]2.0.CO;2

Leonard, J.A., Wayne, R.K., Wheeler, J., Valadez, R., Guillen, S., VILA, C. 2002. Ancient DNA evidence for old world origin of new world dogs. Science 298(5598): 1643-1616. DOI: 10.1126/science.1076980

LIMA, S.L., BednekofF, P.A. 1999. Temporal variation in danger drives antipredator behavior: The predation risk allocation hypothesis. The American Naturalist 153(6): 649-659. DOI: $10.1086 / 303202$

Lowry, D.A., McARThUR, K.L. 1978. Domestic dogs as predator on deer. Wildlife Society Bulletin 6: 38-39.

LUNA, D. 2005. Los humedales no pueden esperar. Manual para el uso racional del sistema de humedales costeros de
Coquimbo. Corporación Ambientes Acuáticos de Chile (CAACH). Coquimbo. 79 pp.

Manly, B., McDonald, L., Thomas, D., McDonald, T., Erickson, W. 2002. Resource selection by animals. Kluwer Academic Publichers, New York. 222 pp.

MANOR, R., SALTZ, D. 2004.The impact of free-roaming dogs on gazelle kid/female ratio in a fragmented area. Biological Conservation 119(2): 231-236. DOI: 10.1016/j. biocon.2003.11.005

MeCH, L.D. 1970. The Wolf: The Ecology and Behaviour of an Endangered Species. Natural History Press, New York. $384 \mathrm{pp}$.

Mills, M.G., BroomhalL, L.S., DU ToIt, J.T. 2004. Cheetah Acininyx jubatus feeding ecology in the Kruger National Park and a comparison across African savanna habitats: is the cheetah only a successful hunter on open grassland plains?. Wildlife Biology 10(3): 177-186. DOI: 10.2981/ wlb.2004.024

MMA. 2016. Lista de especies según estado de conservación. Ministerio de Medio Ambiente. URL: http://www.mma. gob.cl/clasificacionespecies. Accedido: Junio 21, 2016.

MolinA, P.C. 2015. Propuesta de Plan de Manejo para la Avifauna del humedal costero y urbano "El Culebrón". Tesis de Magíster. Universidad Católica del Norte, Coquimbo, Chile.

Pole, A., Gordon, I.J., Gormasn, M.L., McAskill, M. 2004. Prey selection by African wild dogs (Lycaon pictus) in suthern Zimbabwe. Journal of Zoology 262(2): 207-215. DOI: $10.1017 /$ S0952836903004576

R CORE TEAM. 2015. R: A language and environment for statistical computing. $\mathrm{R}$ version 3.2.2. The $\mathrm{R}$ Foundation for Statistical Computing, Vienna, Austria. URL: https:// www.R-project.org.

RADLOFF, F.G., DU TOIT, J.T. 2004. Large predators and their prey in a southern African savanna: a predator's size determines its prey size range. Journal of Animal Ecology73(3): 410423. DOI: 10.1111/j.0021-8790.2004.00817.x

Roley, A., Gormley, A., Forsyth, D.M., Wilton, A.N., Stephens, D. 2010. Movements and habitat selection by wild dogs in eastern Victoria. Australian Mammalogy 32(1): 23-32. DOI: 10.1071/AM09030

RSPCA (s/f). Operational guidance for dog-control staff. The Royal Society for the Prevention of Cruelty to Animals, United Kingdom. 19 pp.

SAG. 2015. Ley de caza y su reglamento. División de protección de los recursos naturales renovables. Servicio Agrícola y Ganadero, Santiago de Chile. 110 pp.

Savolainen, P., Zhang, Y., Luo, J., Lundeberg, J., Leitner, T. 2002. Genetic evidence for East Asian origin of domestic dogs. Science 298(5598): 1610-1613. DOI: 10.1126/ 
science.1073906

Seaby, R.M.H., Henderson, P.A. 2006. Species diversity and richness version 4.1. Lymington, England. 123 pp.

Serpell, J. 1996. The Domestic Dog: Its Evolution, Behaviour and Interactions with People. Cambridge University Press, Cambridge. 284 pp.

Sillero-Zubiri, C., King, A.A. \& Macdonald, D.W. 1996. Rabies and mortality in Ethiopian wolves (Canis simensis). Journal of Wildlife Diseases 32(1):80-86. DOI: 10.7589/00903558-32.1.80

Silva-RodrígueZ, E.A., Sieving, K.E. 2011. Influence of care of domestic carnivores on their predation on vertebrates. Conservation Biology 25(4): 808-815. DOI: 10.1111/j.1523-1739.2011.01690.x

Silva-Rodríguez, E.A., Verdugo, C., Aleuy, O. A., Sanderson, J.G., Ortega-Solis, G.R., Osorio-Zuñiga, F., Gonzalez-Acuña, D. 2010. Evaluating mortality sources for the Vulnerable pudu Pudu puda in Chile: implications for the conservation of a threatened deer. Oryx 44(1): 97-103. DOI: 10.1017/ S0030605309990445

SIME, C. 1999. Domestic dogs in wildlife habitats. En: Joslin, G. Youmans, $H$. (Eds) Effects of recreation on rocky mountain wildlife: 8.1-8.17. Wildlife Society, Colorado.

TABORSKY, M. 1988. Kiwis and dog predation: observations in Waitangi State Forest. Notornis 35(3): 197-202.
VAnak, A.T., Gompper, M.E. 2009a. Dogs Canis familiaris as carnivores: their role and function in intra guild competition. Mammal Review 39(4): 265-283. DOI: 10.1111/j.1365-2907.2009.00148.x

VAnAK, A.T., Gompper, M.E. 2009b. Dietary niche separation between sympatric free-ranging domestic dogs and Indian foxes in central India. Journal of Mamalogy 90(5): 1058-1065. DOI: 10.1644/09-MAMM-A-107.1

Weston, M.A., Fitzsimons, J.A., Wescott. G., Miller, K.K., Ekanayake, K.B., Schneider, T. 2014. Bark in the park: a review of domestic dogs in parks. Environmental Management 54(3): 373-382. DOI: 10.1007/s00267-014-0311-1

Wierzbowska, I., Hedrzak, M., Popczyk, B., Crooks, K.R. 2016. Predation of wildlife by free-ranging domestic dogs in Polish hunting grounds and potential competition with the grey wolf. Biological Conservation 201: 1-9. DOI: 10.1016/j.biocon.2016.06.016

Wikelski, M., Foufopoulos, J., Vargas, H., Snell, H. 2004. Galápagos birds and diseases: Invasive pathogens as threats for island species. Ecology and Society 9(1): 5. DOI: 10.5751/ ES-00605-090105

Yalden, P.E., Yalden. D. 1990. Recreational disturbance of breeding golden plovers Pluvialis apricarius. Biological Conservation 51(4): 243-262. DOI: 10.1016/00063207(90)90111-2

ANEXo 1. Códigos asignados por especie para la muestra de la ausencia de ataque (0). Código para sitio: 1: interior; 2: playa. Código para sustrato: 1: agua superficial; 2: vegetación. / Codes assigned by species for the sample of the absence of attack (0). Code for site: 1 : inside; 2: beach. Code substrate: 1: surface water; 2: vegetation.

\begin{tabular}{clccc}
\hline $\mathbf{N}^{\circ}$ & \multicolumn{1}{c}{ Especie } & Tamaño $(\mathrm{cm})$ & Sitio & Sustrato \\
\hline 1 & Anas georgica & 51 & 1,2 & 1,2 \\
2 & Anas flavirostris & 41 & 1,2 & 1,2 \\
3 & Anas sibilatrix & 51 & 1,2 & 1,2 \\
4 & Pelecanus thagus & 127 & 1,2 & 1,2 \\
5 & Phalacrocorax brasilianus & 75 & 1,2 & 1,2 \\
6 & Casmerodius albus & 85 & 1,2 & 1,2 \\
7 & Egretta thula & 50 & 1,2 & 1,2 \\
8 & Ardea cocoi & 120 & 1,2 & 1,2 \\
9 & Bubulcus ibis & 50 & 1,2 & 1,2 \\
10 & Nycticorax nycticorax & 65 & 1 & 2 \\
11 & Plegadis chihi & 56 & 2 & 1,2 \\
12 & Cathartes aura & 81 & 2 & 2 \\
13 & Coragyps atratus & 74 & 1,2 & 2 \\
14 & Caracara plancus & 58 & 2 & 2 \\
15 & Fulica armillata & 51 & 1,2 & 1,2 \\
\hline
\end{tabular}




\begin{tabular}{llccc}
\hline $\mathbf{N}^{\circ}$ & \multicolumn{1}{c}{ Especie } & Tamaño $(\mathrm{cm})$ & Sitio & Sustrato \\
\hline 16 & Fulica leucoptera & 43 & 1,2 & 1,2 \\
17 & Haematopus palliatus & 44 & 1,2 & 2 \\
18 & Himantopus melanurus & 42 & 1,2 & 1,2 \\
19 & Vanellus chilensis & 37 & 1,2 & 2 \\
20 & Charadrius collaris & 16 & 2 & 2 \\
21 & Charadrius modestus & 22 & 2 & 2 \\
22 & Tringa melanoleuca & 33 & 1,2 & 1,2 \\
23 & Calidris canutus & 26 & 2 & 2 \\
24 & Numenius phaeopus & 42 & 2 & 1,2 \\
25 & Chroicocephalus maculipennis & 38 & 1,2 & 1 \\
26 & Larus dominicanus & 62 & 1,2 & 1,2 \\
27 & Rynchops niger & 46 & 2 & 2 \\
28 & Columbia livia & 36 & 2 & 1,2 \\
29 & Cinclodes fuscus & 18 & 1,2 & 2 \\
30 & Muscisaxicola maclovianus & 16 & 2 & 2 \\
31 & Lessonia rufa & 13 & 2 & 2 \\
32 & Tachuris rubrigastra & 10,5 & 1,2 & 1,2 \\
33 & Tachycineta meyeni & 14 & 1,2 & 2 \\
34 & Cistothorus platensis & 11 & 1,2 & 2 \\
35 & Agelaius thilius & 20 & 1,2 & 1 \\
36 & Sturnella loyca & 28 & & 2 \\
\hline & & & 2 & \\
\hline
\end{tabular}

Received: 11.05 .2018

Accepted: 26.04.2019 